\title{
Desafío diagnóstico y terapéutico de carcinoma mucoepidermoide palatino: reporte de un caso
}

\section{Diagnostic and therapeutic challenge in palatal mucoepidermoid carcinoma: a case report}

\author{
Valentina Vergara $G .{ }^{1}$, Nicole Sabelle $H .{ }^{2}$, Iris Espinoza S. ${ }^{5}$, Marcelo Mardones M. ${ }^{1,3}$, \\ Cristóbal Araya S. ${ }^{4}$, Andrea Maturana R. ${ }^{5,6}$
}

'Departamento de Cirugía y Traumatología Bucal y Máxilofacial, Facultad de Odontología, Universidad de Chile. Santiago, Chile.

Escuela de Pregrado, Facultad de Odontología de la Universidad de

Chile. Santiago, Chile.

${ }^{3}$ Servicio de Cirugía Oral y Maxilofacial, Hospital San José. Santiago, Chile.

${ }^{4}$ Servicio de Cirugía de Cabeza y Cuello, Instituto Nacional del Cáncer. Santiago, Chile.

${ }^{5}$ Departamento de Patología y Medicina Oral, Facultad de Odontología de la Universidad de

Chile. Santiago, Chile.

${ }^{6}$ CDT Eloísa Díaz Hospital San José, Dental/Patología y Medicina Oral. Servicio de Anatomía Patológica, Hospital San José. Santiago, Chile.

Los autores declaran no tener conflictos de interés. Se agradece al equipo de Cirugía de Cabeza y Cuello del Instituto Nacional del

Cáncer y a la Fundación OREMA quienes participaron en el tratamiento oncológico y reconstructivo del paciente.

Recibido el 6 de julio de 2020. Aceptado el 1 de octubre de 2020.

Correspondencia: Andrea Maturana R. Olivos 943, Independencia. Código Postal: 8380492. Santiago, Chile. Email: amaturana@odontologia. uchile.cl

\section{Resumen}

Las neoplasias en glándulas salivales son infrecuentes, representando menos del $3 \%$ de los tumores de cabeza y cuello. El carcinoma mucoepidermoide es el tumor maligno más común en glándulas salivales, siendo su principal ubicación la parótida. Clínicamente se asemeja a otras lesiones de mucosa oral, por lo cual, es importante realizar un correcto diagnóstico diferencial. Su comportamiento biológico se relaciona con el grado histológico tumoral, factor relevante en el pronóstico y tratamiento de esta neoplasia. Presentamos el caso de un paciente hombre de 75 años afectado con un tumor en paladar con diagnóstico de carcinoma mucoepidermoide de bajo grado. Como tratamiento se realizó una maxilectomía parcial y una placa obturadora en base a una prótesis removible y posterior reconstrucción con un colgajo libre microvascularizado. Actualmente el paciente se encuentra en controles periódicos, libre de enfermedad. Los tumores de glándulas salivales son un desafío diagnóstico, requieren de exámenes imagenológicos y del estudio histopatológico. Cuando existen dudas en el diagnóstico, se debe considerar repetir la toma de la muestra o la obtención de biopsias de más de una zona representativa que permita el diagnóstico de la lesión.

Palabras clave: tumor de glándulas salivales, carcinoma mucoepidermoide, glándula salival menor, neoplasias, paladar.

\section{Abstract}

Salivary gland neoplasms are infrequent lesions representing less than 3\% of head and neck tumors. Mucoepidermoid carcinoma is the most common malignant tumor in salivary glands, being the parotid the most usual location. Clinically, it resembles other oral mucosa lesions, therefore, it is important to make a correct differential diagnosis. Its biological behavior is related to the tumor histological grade, a relevant factor in the prognosis and treatment of this neoplasm. We reported a case of a 75-year-old-man, with a tumor in the palate, diagnosed as low-grade mucoepidermoid carcinoma. A partial maxillectomy and an obturator plate were performed based on a removable prosthesis and subsequent reconstruction with a microvascularized free flap. The patient is currently undergoing regular check-ups, maintaining disease free. Salivary gland tumors are a diagnostic challenge, requiring imaging tests and histopathological study. In case of doubts with the diagnosis, it should be considered to biopsy more than area or to repeat the biopsy in order to obtain a representative sample that allows the diagnosis of the lesion.

Keywords: Salivary gland tumor, mucoepidermoid carcinoma, minor salivary glands, neoplasms, palate. 


\section{Introducción}

Las neoplasias de glándulas salivales (GS) son lesiones poco frecuentes, comprenden solo entre el 3\% y $6 \%$ de los tumores de cabeza y cuello ${ }^{1}$. El carcinoma mucoepidermoide (CME) es el tumor maligno más común de $\mathrm{GS}^{2}$, corresponde entre el $4 \%$ al $10 \%$ de todos los tumores de GS mayores y entre el 13\% al 23\% de GS menores, siendo en este último grupo, el paladar el sitio más frecuente ${ }^{3}$. Respecto a la edad, afecta desde la segunda a séptima década de vida $^{2,3}$. En Chile, un estudio en Valparaíso determinó que el CME correspondía al 7,7\% del total de neoplasias benignas y malignas de GS ${ }^{4}$.

Histológicamente, el CME presenta cantidades variables de células epidermoides, intermedias y mucosas, y es caracterizado en grados de severidad según presencia de necrosis, invasión linfática, invasión a tejido óseo, porcentaje de mitosis y presencia de componente quístico, siendo este último, un importante indicador de grado de malignidad ${ }^{2,5,7}$. Clínicamente se presentan, inicialmente, como tumoraciones asintomáticas de variada induración y consistencia, pudiendo ser fluctuantes según el componente quístico y de coloración rojo y azulado, por lo que pueden confundirse clínicamente con otras lesiones ${ }^{3,6}$ (Tabla 1). A continuación, se presenta el reporte de un caso clínico realizado bajo la pauta CARE ${ }^{8}$.

\section{Caso Clínico}

Paciente masculino, 75 años, sin antecedentes mórbidos ni alergias. Consulta en el Servicio de Patología Oral del Hospital San José, por presentar un tumor de 2 meses de evolución, refiere dolor 5/10 en escala visual análoga, esporádico, que se intensifica al comer. Al examen intraoral, es desdentado parcial bimaxilar y portador de prótesis parcial removible. A nivel palatino se observa tumor de $3 \mathrm{~cm} \mathrm{x} 2 \mathrm{~cm}$, en límite de paladar duro y blando del lado derecho, ovalado, cubierto por mucosa rosada con zonas centrales de coloración azulada, base sésil, fluctuante y doloroso a la palpación (Figura 1A). Se solicita tomografía computarizada (TC) de senos paranasales, en corte sagital y coronal, donde se observa crecimiento expansivo hacia piso de fosa nasal con adelgazamiento de la cortical (Figura 1B).

Tabla 1. Diagnósticos diferenciales de caso clínico reportado (Tabla adaptada de libro: Neville y cols. ${ }^{3}$ )

\begin{tabular}{|c|c|c|}
\hline Lesiones & Características clínicas & $\begin{array}{l}\text { Localización más frecuente en } \\
\text { cavidad oral }\end{array}$ \\
\hline Absceso palatal & $\begin{array}{l}\text { Aumento de volumen de consistencia } \\
\text { fluctuante asociado con diente no } \\
\text { vital o con enfermedad periodontal } \\
\text { avanzada }\end{array}$ & Mucosa de zona palatina de molares \\
\hline $\begin{array}{l}\text { Tumores de glándulas salivales } \\
\text { (especialmente adenoma pleomórfico, } \\
\text { carcinoma adenoide quístico, } \\
\text { adenocarcinoma polimorfo) }\end{array}$ & $\begin{array}{l}\text { Tumor de consistencia blanda o firme, } \\
\text { pueden tener color similar a mucosa } \\
\text { normal o un tono azulado }\end{array}$ & Paladar \\
\hline $\begin{array}{l}\text { Hiperplasia adenomatoide de } \\
\text { glándulas salivales menores }\end{array}$ & $\begin{array}{l}\text { Tumor de base sésil, indoloro, blando } \\
\text { o firme a la palpación, con color } \\
\text { similar a mucosa normal o tono rojo } \\
\text { o azulado }\end{array}$ & Paladar duro o blando \\
\hline Neurofibroma & $\begin{array}{l}\text { Tumor de consistencia blanda a firme, } \\
\text { cubierto por mucosa de aspecto } \\
\text { normal }\end{array}$ & Lengua y mucosa bucal \\
\hline Mucocele/quiste del conducto salival & $\begin{array}{l}\text { Tumor de consistencia blanda y/o } \\
\text { fluctuante, puede tener tono azulado } \\
\text { o color similar a mucosa normal }\end{array}$ & $\begin{array}{l}\text { Mucosa de zona de labio inferior y } \\
\text { paladar (raro) }\end{array}$ \\
\hline
\end{tabular}


Se indica biopsia incisional. Durante el acto quirúrgico de obtención de la biopsia, posterior a la incisión mucosa, destaca el drenaje de contenido líquido incoloro. La muestra fue insuficiente para diagnóstico histopatológico, por lo que se realiza segunda biopsia incisional. En el segundo informe histopatológico, se describen múltiples zonas quísticas delimitadas por epitelio en su mayor parte cúbico, de 2-3 capas, con tendencia a engrosarse y a formar proyecciones papilares hacia el lumen, toda la muestra presentaba abundante proceso inflamatorio crónico (Figura 1C-E). Mediante inmunohistoquímica se pueden evaluar islotes y cordones de células epiteliales distantes, adyacentes a tejido óseo vital. Con tinción de azul alcian se destaca la presencia de células mucosas (Figura 1F). Con tinción S100 se

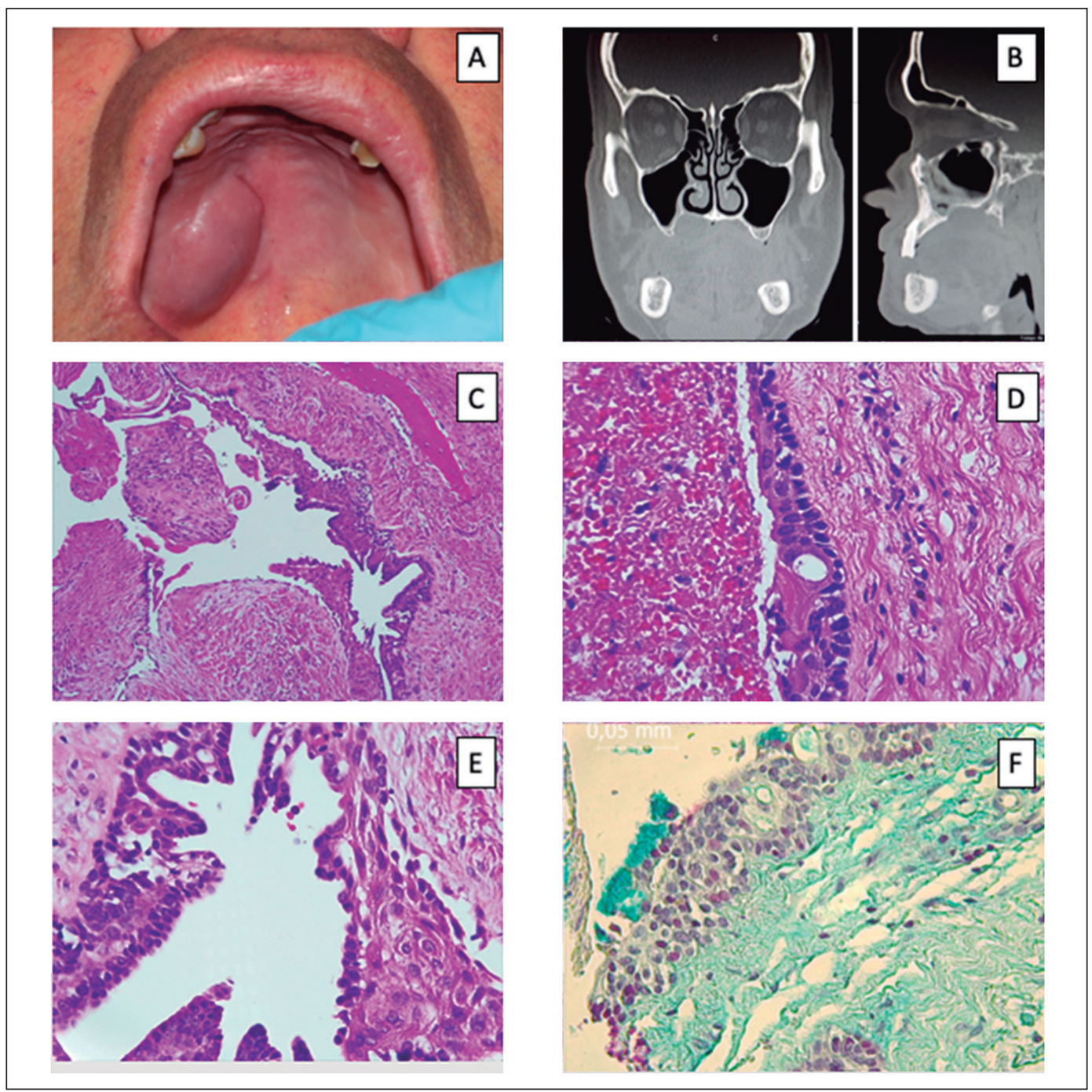

Figura 1. A: Imagen clínica intraoral tumor ubicado en paladar duro y blando, lado derecho, superficie lisa, cubierto por mucosa de aspecto y coloración normal, pero que hacia la línea media posterior cambia a un color levemente azulado derecho. B: Tomografía computarizada corte coronal y axial que evidencia masa tumoral que adelgaza la cortical ósea adyacente a la lesión geniana. C: Proliferación de epitelio que delimita parcialmente una cavidad quística cercana a trabécula de tejido óseo vital inmerso en tejido fibroso 10x en tinción hematoxilina-eosina. D: Epitelio delgado de 2 a 3 capas de grosor 40x en tinción hematoxilina-eosina. E: Epitelio con formación de proyecciones papilares hacia el lumen ductal y células cúbicas y ciliadas en la superficie, además, se aprecia que el epitelio sufre cambio de tipo escamoso 40x en tinción hematoxilina-eosina. F: Zonas de engrosamiento epitelial con células claras y células mucosecretoras en la superficie 40x en tinción azul alcian. 


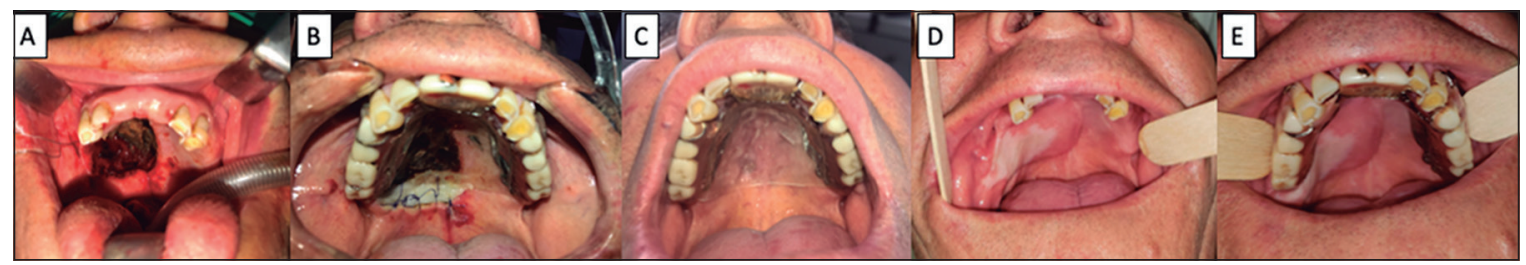

Figura 2. Cirugía reconstructiva. A: Primera cirugía reconstructiva, maxilectomía parcial derecha. B: Primera cirugía reconstructiva, confección de placa obturadora en base a la prótesis removible en forma de herradura. C: Primera cirugía reconstructiva, se fija con alambre a proceso alveolar. D: Segunda cirugía reconstructiva, reconstrucción con colgajo libre microvascular radial. E: Segunda cirugía reconstructiva, se modifica el obturador y se instala a modo de prótesis removible.

detectó una marcación del 10\% en células neoplásicas, Ki67 positividad cercana al 20\% en células neoplásicas, miosina de músculo liso - cadena pesada (SMM-HC) positiva en células mioepiteliales neoplásicas y mamoglobina fue negativa. El diagnóstico fue de neoplasia de glándulas salivales sugerente de cistoadenoma, con indicación de analizar la muestra por completo para el diagnóstico definitivo. No se podía descartar CME de bajo grado.

Se deriva paciente al Servicio de Cirugía de Cabeza y Cuello del Instituto Nacional del Cáncer de Chile. El estudio imagenológico mediante TC de senos paranasales, cuello y tórax, señala tumor de paladar con aspecto quístico y adelgazamiento de piso de fosas nasales, sin compromiso cervical ni torácico. Se planifica maxilectomía parcial derecha e instalación de placa obturadora con adaptación de prótesis dental removible del paciente más fijación alámbrica a proceso alveolar remanente (Figura 2A-C). La biopsia diferida y definitiva establece un diagnóstico de CME de bajo grado, sin márgenes comprometidos. El comité oncológico determina seguimiento por un periodo de 6 meses, estudio imagenológico para etapificación y posterior reconstrucción con colgajo libre microvascular radial (Figuras $2 \mathrm{D}$ y $2 \mathrm{E})$.

\section{Discusión}

En el presente artículo exponemos el caso de un hombre con un tumor de paladar, cuyo diagnóstico definitivo fue CME de bajo grado. A diferencia de las GS mayores, en donde reparos anatómicos con el nervio facial tornan compleja la obtención de una muestra, en cavidad oral, se puede acceder sin mayores complicaciones para profesionales debidamente entrenados. En estos casos la indicación es biopsia incisional, puesto que cerca del $41 \%$ de tumores de GS menores son neoplasias malignas ${ }^{9}$. Es importante considerar el diagnóstico clínico diferencial con otras lesiones orales como quistes de tejidos blandos, abscesos odontogénicos, neoplasias mesenquimáticas y otros tumores de $\mathrm{GS}^{2,3,5,10}$ (Tabla 1).

El dolor relatado por los pacientes en este tipo de lesiones podrían asociarse con un posible compromiso e invasión perineural descrito en lesiones como el carcinoma adenoideo quístico. En el CME, los pacientes pueden relatar sintomatología dolorosa cuando existe compromiso de vainas nerviosas y parestesia y/o parálisis del nervio facial cuando el tumor afecta la parótida ${ }^{3}$. Sin embargo, en el caso presentado no se observó compromiso neural en el análisis microscópico y pudo ser por compresión constante del tejido al momento de alimentarse.

Histológicamente, los CME son categorizados según la cantidad de zonas de formación quística, grado de atipia celular, número relativo de células mucosas, epidermoides e intermedias, zonas de necrosis, compromiso óseo, vascular y perineural en bajo, intermedio y alto grado. Los CME de bajo grado, muestran tendencia a la formación de estructuras quísticas, atipia celular mínima y proporción relativamente alta de células mucosas; siendo su tratamiento la resección como en el caso presentado. El estudio imagenológico es fundamental para evaluar compromiso cervical, siendo poco frecuente la de disección cervical. CME de alto grado constan de islas sólidas de células escamosas e intermedias, considerable 
pleomorfismo y actividad mitótica. El grado intermedio muestra características de los dos grados anteriormente nombrados. Para el tratamiento de los CME de grado intermedio y alto, se indica resección oncológica con estudio mediante biopsia rápida, con o sin disección cervical. La AJCC recomienda el manejo cervical similar a los carcinomas escamosos orales, sin embargo, las principales consideraciones sobre disecar los niveles ganglionares comprometidos consideran parámetros clínicos como presencia de linfonodos en el examen físico, estudio por imágenes, extensión y compromiso óseo y grado histológico ${ }^{2,3,5,11-13}$.

Las tasas de supervivencia global a los 10 años de los CME de bajo, intermedio y alto grado, corresponden a un $90 \%, 70 \%$ y $25 \%$ respectivamente $^{2}$. En este reporte, la biopsia incisional inicial fue insuficiente y requirió una segunda muestra para establecer el diagnóstico, por lo que se sugiere siempre tomar una muestra representativa con un tamaño y profundidad adecuada basados en la hipótesis diagnóstica clínica. Las características histológicas encontradas como proyecciones papilares en cavidades quísticas y leve pleomorfismo celular con ausencia de un patrón de invasión neoplásico clásico, orientaron inicialmente al diagnóstico de un cistoadenoma. La abundante inflamación presente en la biopsia impidió un adecuado diagnóstico, generando dificultad para diferenciar esta lesión entre un cistoadenoma y un CME de bajo grado. Es importante destacar que la OMS desde el año 2017 eliminó entre sus diagnósticos el cistoadenocarcinoma, el que podría haber correspondido al diagnóstico diferencial de esta neoplasia.

El CME de bajo grado tiene bajo riesgo de metástasis regional y a distancia, por lo que su tratamiento corresponde a resección quirúrgica oncológica, con margen de seguridad, sin requerir de terapias adyuvantes como radioterapia o quimioterapia, con buen pronóstico y sin presentar recidivas, en un $90 \%$ al $98 \%$ de los pacientes solo con la cirugía de resección ${ }^{3}$.

Frecuentemente, los tratamientos de tumores malignos de GS de paladar generan grandes secuelas, tanto físicas como funcionales, siendo la rehabilitación del paciente un gran desafío. Se han descrito en la literatura diferentes tratamientos, los que incluyen desde la cicatrización por segunda intención en defectos menores, colgajos para cubrir el defecto, hasta la fabricación de un obturador. En el caso presentado se utilizó la prótesis removible maxilar del paciente, modificándola como un obturador, durante el periodo de seguimiento inicial, previo a la reconstrucción definitiva, mediante un colgajo libre radial microvascular, que permite una separación anatómica y funcional entre cavidad nasal y cavidad oral, facilitando respiración, fonación y alimentación, fundamental para mejorar la calidad de vida del paciente ${ }^{12,14}$.

\section{Conclusión}

El CME corresponde al tumor maligno de GS más frecuente, el cual debe ser considerado como posible diagnóstico diferencial en tumoraciones en zonas de GS mayores y menores. Es importante considerar que múltiples intervenciones pueden dificultar el proceso diagnóstico al generar procesos inflamatorios que alteran la arquitectura histopatológica característica del tejido, por lo que es fundamental realizar una biopsia inicial adecuada, considerando las características clínicas e imagenológicas que presente la lesión, lo que permitirá un diagnóstico histopatológico certero. El uso del obturador con la prótesis del paciente, puede ser una solución efectiva y de bajo costo en este caso, facilitando la alimentación y comunicación en el periodo previo a la reconstrucción, sin embargo, no debe ser considerada como una solución definitiva, pues se encuentra lejos del estándar actual de calidad de vida, a diferencia de la cirugía reconstructiva con colgajos libres que permite un tratamiento definitivo para defectos anatómicos severos.

\section{Bibliografía}

1. Bell D, Hanna EY. Salivary gland cancers: biology and molecular targets for therapy. Curr Oncol Rep. 2012;14(2):166-174. doi: 10.1007/s11912-012-0220-5.

2. El-Naggar AK, Chan JKC, Grandis, JR, Takata T, Slootweg PJ. WHO Classification of Head and Neck Tumours. 4a ed. Lyon, France: International Agency for Research on Cancer, 2017:163-164.

3. Neville, BW, Damm, DD, Allen, CM, Chi, AC. Oral and Maxillofacial Pathology. 4a ed, Canadá: Elsevier, 2016:454-457. 
4. Araya J, Martínez R, Niklander S, Marshall M, Esguep A. Incidence and prevalence of salivary gland tumours in Valparaiso, Chile. Med Oral Patol Oral Cir Bucal. 2015;20(5):e532-e539. doi: 10.4317/ medoral.20337.

5. Luna MA. Salivary mucoepidermoid carcinoma: revisited. Adv Anat Pathol. 2006;13(6):293-307. doi: 10.1097/01.pap.0000213058.74509.d3.

6. Kolude B, Lawoyin JO, Akang EE. Mucoepidermoid carcinoma of the oral cavity. J Natl Med Assoc. 2001;93(5):178-184.

7. Ozawa H, Tomita T, Sakamoto K, et al. Mucoepidermoid carcinoma of the head and neck: clinical analysis of 43 patients. Jpn J Clin Oncol. 2008;38(6):414-418. doi: 10.1093/jjco/hyn045

8. Gagnier JJ, Kienle G, Altman DG, et al. The CARE guidelines: consensus-based clinical case report guideline development. J Diet Suppl. 2013;10(4):381390.

9. Jones AV, Craig GT, Speight PM, et al. The range and demographics of salivary gland tumours diagnosed in a UK population, Oral Oncol. 2008;44:407-417.

10. García-Molina D, Castillo JL, Navarro I, Sánchez R,
Burgueño M. Low grade mucoepidermoid carcinoma. Differential diagnosis of masses in palate. Rev Esp Cirug Oral y Maxilofac. 2014;36(3):136-137.

11. Bai S, Clubwala R, Adler E, et al. Salivary mucoepidermoid carcinoma: a multi-institutional review of 76 patients. Head Neck Pathol. 2013;7(2):105-112. doi: 10.1007/s12105-012-0405-0.

12. Kim SM, Park MW, Cho YA, Myoung H, Lee $\mathrm{JH}$, Lee SK. Modified functional obturator for the consideration of facial growth in the mucoepidermoid carcinoma pediatric patient. Int J Pediatr Otorhinolaryngol. 2015;79(10):1761-1764. doi: 10.1016/j.ijporl.2015.06.031.

13. Lydiatt WM, Patel SG, O'Sullivan B, et al. Head and Neck cancers-major changes in the American Joint Committee on cancer eighth edition cancer staging manual. CA Cancer J Clin. 2017;67(2):122-137. doi: $10.3322 /$ caac. 21389 .

14. Baumgardt C, Günther L, Sari-Rieger A, Rustemeyer J. Mucoepidermoid carcinoma of the palate in a 5-year-old girl: case report and literature review. Oral Maxillofac Surg. 2014;18(4):465-469. doi: 10.1007/ s10006-014-0461-6. 